\title{
Effect of ascorbic acid-rich diet on in vivo-induced oxidative stress
}

\author{
Renata Alleva ${ }^{1 *}$, Ferruccio Di Donato ${ }^{2}$, Elisabetta Strafella ${ }^{3}$, Sara Staffolani ${ }^{3}$, Linda Nocchi $^{3}$, \\ Battista Borghi ${ }^{1}$, Elettra Pignotti ${ }^{4}$, Lory Santarelli ${ }^{3}$ and Marco Tomasetti ${ }^{3}$ \\ ${ }^{1}$ Department of Anesthesiology Research Unit, IRCCS Rizzoli Orthopaedic Institute, Via Pupilli, 1-40136 Bologna, Italy \\ ${ }^{2}$ Hyperbaric Therapy Centre, MPM, Via Tranquillo Cremona, 8, 40137 Bologna, Italy \\ ${ }^{3}$ Department of Molecular and Clinic Science, Polytechnic University of Marche, Via Tronto, 10-60126 Ancona, Italy \\ ${ }^{4}$ Department of Statistical Science, University of Bologna, Via delle Belle Arti, 41 Bologna, Italy
}

(Submitted 14 December 2010 - Final revision received 21 July 2011 - Accepted 21 July 2011 - First published online 16 September 2011)

\section{Abstract}

Using hyperbaric oxygen (HBO) therapy as an in vivo oxidation model, we investigated the effect of a diet enriched in ascorbic acid (AA) on HBO-induced oxidative stress. Volunteers ( $n$ 46) were allocated to the AA-rich diet group or the control group. Blood samples were collected at the basal time, after the 1-week diet before and immediately after the HBO treatment, and 1 week after the HBO treatment. AA level, total antioxidant status (TAS), hydroperoxides (HP), lymphocyte DNA oxidation and DNA repair capacity were assessed. The expression of genes involved in oxidative stress was evaluated in lymphocytes and the protein activity of the modulated genes was determined in the plasma. The AA level and the antioxidant status of plasma were increased by AA-rich food consumption. HBO exposure did not affect the AA levels or TAS, but induced HP formation in the control group. The lymphocytes isolated from dietary-supplemented subjects were resistant to ex vivo DNA oxidation, showing an increased DNA repair capacity compared with controls. A difference in gene expression pattern was observed between the groups. AA-rich foods provide dual protection against oxidative stress, enhancing plasma antioxidant levels and stimulating genes involved in cell detoxification.

Key words: Ascorbic acid: Diet intake: Oxidative stress: Hyperbaric oxygen therapy: Gene expression

Dietary habits are a major aspect of people's lifestyles that influence health, morbidity and mortality for a range of conditions $^{(1-4)}$. Nutrition and dietary patterns have been shown to have a direct impact on the health of the population and selected patient groups ${ }^{(5)}$. The beneficial effects have been attributed to the reduction of oxidative damage caused by a normal or excessive production of free radicals. Dietary supplementation with antioxidant vitamins seems to be the logical answer to decreasing serum antioxidant concentrations, but antioxidants may have adverse effects. A large number of primary or secondary prevention randomised trials have been conducted to assess the benefits and harms of antioxidant supplements $v$. placebo or no intervention. Systematic reviews and meta-analyses of these randomised trials have not demonstrated that $\beta$-carotene, vitamin $\mathrm{A}$ and vitamin $\mathrm{E}$ in the administered dosages lead to decreased mortality, and some analyses have suggested the possibility of increased mortality, while for vitamin $\mathrm{C}$, the verdict is still out ${ }^{(6,7)}$. A mixture of vitamin $\mathrm{C}$ and vitamin $\mathrm{E}$ led to a significant decrease in DNA base oxidation $^{(8,9)}$, but in other trials, antioxidants were ineffective ${ }^{(10,11)}$. Some antioxidants may act as pro-oxidants modulating cellular redox status and oxidising redox-sensitive sites. There are several possible explanations for the potential negative effect of antioxidant supplements. Reactive oxygen species (ROS) in moderate concentrations are essential mediators of reactions by which the body gets rid of unwanted cells. Thus, if the administration of antioxidant supplements decreases free radicals, it may interfere with essential defensive mechanisms for ridding the organism of damaged cells, including those that are precancerous and cancerous ${ }^{(12)}$. However, observational studies have shown that there is a positive association between a healthy diet, especially high intake of fruits and vegetables, and delayed ageing, reduced risk of cancer and reduced risk of $\mathrm{CVD}^{(13,14)}$.

In the present study, we investigated the effect of ascorbic acid (AA) supplementation by diet on oxidative stress induced

Abbreviations: AA, ascorbic acid; au, arbitrary units; DNA-SB, DNA strand breaks; GPX, glutathione peroxidase; GST, glutathione S-transferase; HBO, hyperbaric oxygen; HP, hydroperoxides; ROS, reactive oxygen species; RPMI, Roswell Park Memorial Institute; T1, blood samples collected at the basal time; T2, blood samples collected after the 1-week diet before the HBO treatment; T3, blood samples collected immediately after the HBO treatment; T4, blood samples collected 1 week after the HBO treatment; TAS, total antioxidant status.

*Corresponding author: R. Alleva, fax + 39051 6366344/340, email rena.alleva@gmail.com 
in vivo by hyperbaric oxygen (HBO) treatment. $\mathrm{HBO}$, the intermittent exposure of the body to $100 \% \mathrm{O}_{2}$ at a pressure $>1$ atmosphere absolute, has been successfully used to treat various clinical conditions including soft tissue infections, wound healing and carbon monoxide intoxication ${ }^{(15)}$. It has been suggested that the detrimental effects of exposure to high concentrations of $\mathrm{O}_{2}$ can lead to an abundance of ROS, which can cause cellular damage by lipid, protein and DNA oxidation $^{(16-20)}$. Therefore, HBO exposure represents a model to study the in vivo response to oxidative stress ${ }^{(20)}$.

Here, forty-six healthy volunteers were enrolled at the HBO centre and were allocated to the AA-rich diet group $(600 \mathrm{mg}$ $\mathrm{AA} / \mathrm{d}$ introduced by diet) or the reference group. Blood samples were collected at the basal time (T1), after the 1-week diet before (T2) and immediately after the HBO treatment (T3), and 1 week after the HBO treatment (T4). AA plasma level, plasma hydroperoxides (HP), total antioxidant status (TAS), lymphocyte DNA oxidation and DNA repair activity were assessed. Furthermore, the expression of genes involved in oxidative stress was evaluated in the lymphocytes by quantitative RT-PCR array and the protein activity of the modulated genes was determined in the plasma.

\section{Materials and methods}

\section{Ethical statement}

The study was conducted according to the Declaration of Helsinki and the procedures involving human subjects were processed under approval of the Ethics Committee of the IRCCS Rizzoli Orthopaedic Institute, Bologna, Italy. Written informed consent was obtained from all subjects.

\section{Subjects and study design}

The volunteers were contacted by email, asking them to fill in a FFQ to evaluate their lifestyle, dietary habits and energy intake. From March to July 2010, forty-eight subjects (thirtyone men and seventeen women, mean age 46.4 (SD 8.6) years), after giving their informed consent, were enrolled at the Hyperbaric Therapy Centre, MPM, of Bologna, Italy. They were healthy non-smokers and were not taking antioxidant supplementation or medication.

The subjects were enrolled in five sessions of five to ten subjects each and underwent a clinical examination to assess their suitability for the HBO treatment; two subjects were excluded from the study due to pressure compensation problems. Anthropometric parameters (weight, height and BMI) and body composition (osseous mass, lean mass, fat mass, visceral fat mass and water) were measured by an impedanciometry device (TBF 300 GS; TANITA Corporation, Arlington Heights, IL, USA). According to their dietary habits, the subjects were allocated to two groups. Subjects with a diet rich in vegetables were included in group A ( $n$ 23, fifteen males and eight females), and were asked to follow an isoenergetic diet enriched with foods containing AA such as orange juice, red fruits, kiwi and green vegetables equally distributed over the day to introduce $600 \mathrm{mg} \mathrm{AA} / \mathrm{d}$ (AA-enriched diet). Subjects with a diet poor in vegetables were included in group C ( $n$ 23, fourteen males and nine females), and they were requested to maintain their habitudinal diet during the trial.

\section{Sampling and lymphocyte isolation}

Venous blood samples $(10 \mathrm{ml})$ were collected in heparinised tubes at T1, T2, T3 and T4 time points. The blood samples were centrifuged at $1000 \mathrm{~g}$ for $15 \mathrm{~min}$, and the plasma obtained was stored at $-80^{\circ} \mathrm{C}$ for subsequent biochemical analysis. The buffy coat was removed, placed in a $15 \mathrm{ml}$ tube, and suspended in $4 \mathrm{ml}$ of Roswell Park Memorial Institute (RPMI)-1640 medium. The suspension was then layered onto $4 \mathrm{ml}$ Lympholyte-H (Cederlane, Hornby, ON, Canada) and centrifuged at $700 \mathrm{~g}\left(20^{\circ} \mathrm{C}, 30 \mathrm{~min}\right)$. After centrifugation, the cloudy layer was collected and placed in a $15 \mathrm{ml}$ tube, filled with PBS ( $\mathrm{pH} \mathrm{7.4)}$, and centrifuged at $700 \mathrm{~g}\left(20^{\circ} \mathrm{C}\right.$, $5 \mathrm{~min}$ ). After removing the supernatant, the pellet was resuspended in the RPMI-1640 medium supplemented with 10\% fetal bovine serum, and the cells were counted and assessed for viability using the trypan blue exclusion method and assessed for viability before starting the comet assay.

\section{Hyperbaric protocol}

The subjects were exposed to the HBO treatment within 20.00-21.00 hours before dinner, at least $3 \mathrm{~h}$ after the last meal in accordance with a routine therapy protocol. The multiplace chamber (Sistemi Iperbarici, Ardea, Italy) was pressurised with compressed air where the patients breathed $100 \% \mathrm{O}_{2}$ using a mask at a pressure of 2.5 atmosphere absolute for a total of three 25 min sessions, interrupted with 3 min sessions of air breathing.

\section{DNA repair assay}

DNA repair activity was measured in the lymphocytes of the enrolled subjects at the T1, T2, T3 and T4 time points (five to ten subjects per session) using the comet assay (singlecell gel electrophoresis) ${ }^{(21)}$. Immediately after isolation, the lymphocytes were suspended in PBS and DNA strand breaks (DNA-SB) were introduced by incubating them with $100 \mu \mathrm{M}-$ $\mathrm{H}_{2} \mathrm{O}_{2}$ for $5 \mathrm{~min}$ on ice. The cells were then suspended in a twenty-four-well plate with RPMI-1640 medium containing $10 \%$ fetal bovine serum and incubated at $37^{\circ} \mathrm{C}$ for $6 \mathrm{~h}$. At regular interval times $(0,0.5,1,2,4$ and $6 \mathrm{~h})$, aliquots of the samples were collected. The cells were suspended in $80 \mu \mathrm{l}$ of $1 \%$ low-melting-point agarose (Sigma, St Louis, MO, USA) at $37^{\circ} \mathrm{C}$, and placed on a glass microscope slide (precoated with agarose to aid attachment of the gels). Then, two gels were prepared for each sample. The gels were allowed to set at $4^{\circ} \mathrm{C}$, and the cells were lysed overnight in $2.5 \mathrm{M}-\mathrm{NaCl}$, $0 \cdot 1 \mathrm{M}-\mathrm{Na}_{2}$ EDTA, $10 \mathrm{~mm}$-Tris-HCl ( $\mathrm{pH}$ 10) and $1 \%$ Triton $\mathrm{X}-100$ at $4^{\circ} \mathrm{C}$. The slides were immersed in $0.3 \mathrm{M}-\mathrm{NaOH}$ and $1 \mathrm{~mm}-\mathrm{Na}_{2}$ EDTA for $40 \mathrm{~min}$ at $4^{\circ} \mathrm{C}$ before electrophoresis at $0.8 \mathrm{~V} / \mathrm{cm}$ for $30 \mathrm{~min}$. After neutralisation, the gels were stained with $4^{\prime}$,6-diamidine-2'-phenylindole dihydrochloride, and viewed by fluorescent microscopy. Nucleoid DNA 
extends under electrophoresis to form a 'comet tail' and the relative intensity of DNA in the tail reflects DNA break frequency. The amount of strand breaks was assessed with a visual scoring method; 100 comets randomly selected were graded according to the degree of damage into five classes $(0-4)$ to give an overall score for each gel between 0 and 400 arbitrary units (au).

The ability of cells to repair $\mathrm{H}_{2} \mathrm{O}_{2}$-induced strand breaks was determined by evaluating the persistence of DNA damage over time. The kinetics of DNA repair was calculated as a percentage of the basal value, and the rate of DNA repair (slope of the curve), the first-order rate constant $K(1 / \mathrm{min})$, the maximum velocity of repair $V_{\max }\left(\mathrm{au} \times 10^{-3} / \mathrm{min}\right)$ and half-life $t_{1 / 2}(\mathrm{~h})$ were determined using plots of data obtained from the initial time $\left(t_{\mathrm{i}}\right)$ of induced DNA damage level $\left(C_{\mathrm{i}}\right)$ to the final time $\left(t_{\mathrm{f}}\right)$ when damage was completely repaired $\left(C_{\mathrm{f}}\right)$, according to the following formulas: $K=\left(\log C_{\mathrm{i}}-\log C_{\mathrm{f}}\right) /\left(t_{\mathrm{f}}-t_{\mathrm{i}}\right)$, $V_{\max }=K \times C_{\mathrm{i}}$ and $t_{1 / 2}=\log 2 / K$. The area under the curve was also evaluated by the trapezoidal area method and is expressed as au/min.

\section{Ascorbic acid determination}

The plasma content of AA was measured using a spectrophotometric method ${ }^{(22)}$. Briefly, plasma samples were immediately acidified with TCA (5\%) and well mixed by vortex. Next, $13 \mu \mathrm{l}$ of freshly prepared dinitrophenylhydrazine reagent (2\% dinitrophenylhydrazine, $0.25 \%$ thiourea and $0.03 \% \mathrm{CuSO}_{4}$ prepared in $4.5 \mathrm{M}-\mathrm{H}_{2} \mathrm{SO}_{4}$ ) were added to $40 \mu \mathrm{l}$ of acidified plasma. The samples were mixed and incubated at $37^{\circ} \mathrm{C}$ for $6 \mathrm{~h}$. The samples were placed on ice and $50 \mu \mathrm{l}$ of chilled $\left(4^{\circ} \mathrm{C}\right) \mathrm{H}_{2} \mathrm{SO}_{4}(65 \%)$ were added and mixed. The absorbance of the samples was read at $520 \mathrm{~nm}$ in an ELISA plate reader (Sunrise, Tecan, Milan, Italy). The standard curve was generated by dissolving various amounts of AA $(10-500 \mu \mathrm{M})$ in 5\% TCA and treated in the same manner as plasma samples.

\section{Hydroperoxide assay}

HP were evaluated in plasma by the reactive oxygen metabolite (d-ROM) assay ${ }^{(23)}$. Briefly, $3 \mu$ l of plasma were added to a reaction mixture containing $N, N$-diethyl-para-phenylendiamine and acetate buffer ( $\mathrm{pH} 4 \cdot 8$ ). Samples were subjected to repeated spectrophotometric readings $(520 \mathrm{~nm})$ taken over a total of twenty successive analytical spin cycles. The concentration was automatically calculated from the mean slope (the rate of change in absorbance). Data are expressed as a percentage variation with respect to the control (untreated sample).

\section{Total antioxidant status}

The TAS of plasma was evaluated by a commercial kit (RANDOX Laboratories, Antrim, UK) according to the manufacturer's instructions. Incubation of $\mathrm{ABTS}^{\circledR}$ with a peroxidase (metmyoglobin) results in the production of the radical cation ABTS $^{+{ }^{\circledR}}$. This species is blue-green in colour and can be detected at $600 \mathrm{~nm}$. Antioxidants in the added plasma sample cause inhibition of this colour production to a degree that is proportional to their concentration. Data are expressed as Carrell units.

\section{Quantitative RT-PCR array}

Total RNA was extracted from the lymphocytes of five randomly selected subjects from each group using the total RNA purification kit (Versagene; Gentra System, Minneapolis, $\mathrm{MN}$, USA), according to the manufacturer's instructions.

Table 1. Demographic and basic characteristics of the recruited subjects (Mean values and standard deviations)

\begin{tabular}{|c|c|c|c|c|}
\hline \multirow[b]{2}{*}{ Demographic/anthropometric indices } & \multicolumn{2}{|c|}{ Group A } & \multicolumn{2}{|c|}{ Group C } \\
\hline & Mean & SD & Mean & SD \\
\hline$n$ & \multicolumn{2}{|c|}{23} & \multicolumn{2}{|c|}{23} \\
\hline Age (years) & $50 \cdot 9$ & $6 \cdot 9$ & $46 \cdot 8$ & 6.9 \\
\hline Sex (male/female) & \multicolumn{2}{|c|}{$15 / 8$} & \multicolumn{2}{|c|}{$14 / 9$} \\
\hline Weight (kg) & $72 \cdot 5$ & $10 \cdot 5$ & 71.6 & $15 \cdot 7$ \\
\hline Height (cm) & 174.8 & $6 \cdot 3$ & 169.5 & $8 \cdot 3$ \\
\hline $\mathrm{BMI}\left(\mathrm{kg} / \mathrm{m}^{2}\right)$ & 24.6 & $2 \cdot 8$ & $25 \cdot 3$ & 4.7 \\
\hline \multicolumn{5}{|l|}{ Antioxidant/oxidant status } \\
\hline Ascorbic acid $(\mu \mathrm{M})$ & $66 \cdot 7$ & $6 \cdot 4$ & $69 \cdot 2$ & 7.4 \\
\hline Hydroperoxides $\left(\mathrm{OD} / \mathrm{s} \times 10^{-5}\right)$ & $4 \cdot 0$ & 0.5 & $4 \cdot 0$ & 0.8 \\
\hline Total antioxidant status (CU) & $4476 \cdot 3$ & 589.8 & $4700 \cdot 0$ & 1111.6 \\
\hline Glutathione peroxidase (U/l) & $466 \cdot 2$ & $78 \cdot 1$ & $467 \cdot 0$ & $66 \cdot 0$ \\
\hline Glutathione $S$-transferase (U/I) & 13.9 & $11 \cdot 2$ & $16 \cdot 5$ & 11.7 \\
\hline \multicolumn{5}{|l|}{ Body composition $(\mathrm{kg})$} \\
\hline Osseo mass & $2 \cdot 7$ & 0.7 & $2 \cdot 7$ & 0.5 \\
\hline Lean mass & $14 \cdot 7$ & $4 \cdot 2$ & $14 \cdot 2$ & $2 \cdot 2$ \\
\hline Fat mass & $15 \cdot 8$ & $2 \cdot 3$ & $16 \cdot 3$ & $6 \cdot 8$ \\
\hline Visceral fat mass & $7 \cdot 2$ & 4.6 & $7 \cdot 1$ & 3.9 \\
\hline Water & $37 \cdot 7$ & $6 \cdot 0$ & 37.9 & 5.9 \\
\hline
\end{tabular}

OD, optical density; CU, Carrell units. 
The integrity of the RNA obtained was assessed by electrophoresis, and RNA concentration was determined by UV spectrophotometry. Complementary DNA was synthesised using the $\mathrm{RT}^{2}$ First Strand kit (SABiosciences, Frederick, MD, USA) and the expression of eighty-eight genes involved in human oxidative stress and antioxidant defence (PAHS-065A; SABiosciences) was assayed by real-time PCR (Mastercycler ep realplex; Eppendorf, Milano, Italy) using $\mathrm{RT}^{2}$ SYBR Green qPCR Master Mix (SABiosciences).

Genes that were not detected in any samples with a $C_{\mathrm{t}}$ value of $>35$ were excluded from the comparisons. The results are expressed as $C_{\mathrm{t}}$ value normalised to $\beta$-actin $\left(\Delta C_{\mathrm{t}}\right)$ and the comparative $C_{\mathrm{t}}\left(\Delta \Delta C_{\mathrm{t}}\right)$ method was used to determine the fold-change expression. Following log-transformation of the mean ratio of $\Delta \Delta C_{\mathrm{t}}$ for T2:T1, T3:T1, T4:T1 for each gene of groups $\mathrm{A}$ and $\mathrm{C}$, clustering analysis software was used, and the resulting expression map was visualised with Treeview using the average-linkage clustering algorithms (Eisen Lab, Stanford University, CA, USA). Genes with increased expression are indicated in red and those with decreased expression in green. Yellow indicates genes whose expression did not change between T1 and T2, T3 or T4. Black indicates genes that were not detected.

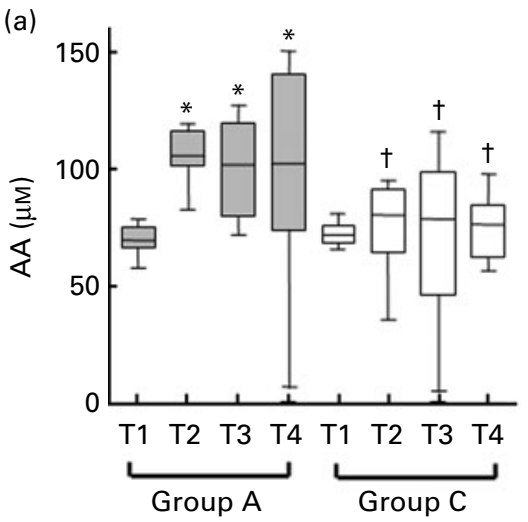

\section{Glutathione peroxidase and glutathione S-transferase assays}

The glutathione peroxidase (GPX) activity of plasma was evaluated by a commercial kit (Trevigen, Gaithersburg, MD, USA), according to the manufacturer's instructions. Plasma samples $(20 \mu \mathrm{l})$ were added to a reaction mixture containing glutathione reductase, glutathione and NADPH. Samples were subjected to repeated UV spectrophotometric readings $(340 \mathrm{~nm})$ taken over a total of fifteen successive analytical spin cycles. GPX activity was automatically calculated from the mean slope (the rate of change in absorbance) using GPX as standard. Data are expressed as U/1.

The glutathione $S$-transferase (GST) activity of plasma was determined by fluorescent commercial kit (ARBOR assays, Ann Arbor, MI, USA), according to the manufacturer's instructions. Briefly, $50 \mu \mathrm{l}$ of diluted plasma samples (1:5) were added to a detection reagent mixture and glutathione. After 30 min of incubation, the fluorescent signal was read in a plate reader (Victor; Perkin Elmer, Boston, MA, USA) at $460 \mathrm{~nm}$ (emission) with excitation at $370 \mathrm{~nm}$. GST activity was calculated on a GST standard curve. Data are expressed as $\mathrm{U} / 1$.

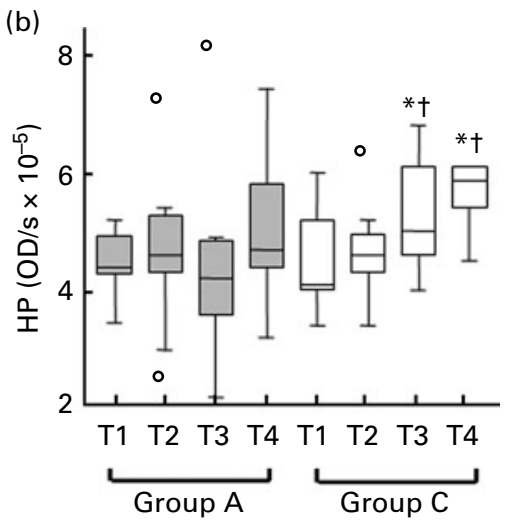

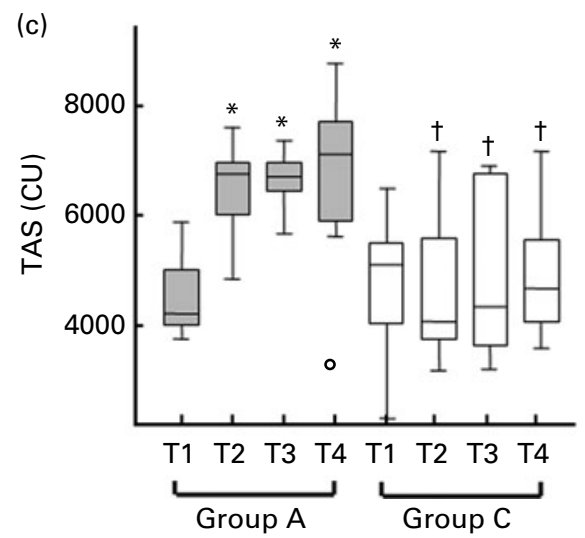

Fig. 1. Box plot showing (a) ascorbic acid (AA), (b) hydroperoxides (HP) and (c) total antioxidant status (TAS) levels in the plasma of diet-supplemented and control subjects undergoing hyperbaric oxygen (HBO) therapy. The levels of AA, HP and TAS were assessed in the plasma of diet-supplemented (group A, $n$ 23) and non-supplemented (group $\mathrm{C}, n$ 23) subjects at the basal time (T1), after the 1-week diet before (T2) and immediately after the HBO treatment (T3) and 1 week after the HBO treatment (T4). The lines in the boxes represent the median values. Lines extend to the minimum and maximum values, excluding the outliers (O). ${ }^{*}$ Mean values were significantly different for T1 v. T2, T3 and T4 $(P<0.05)$. † Mean values were significantly different for group $\mathrm{A} v$. group $\mathrm{C}(P<0.05)$. OD, optical density; CU, Carrell units. 


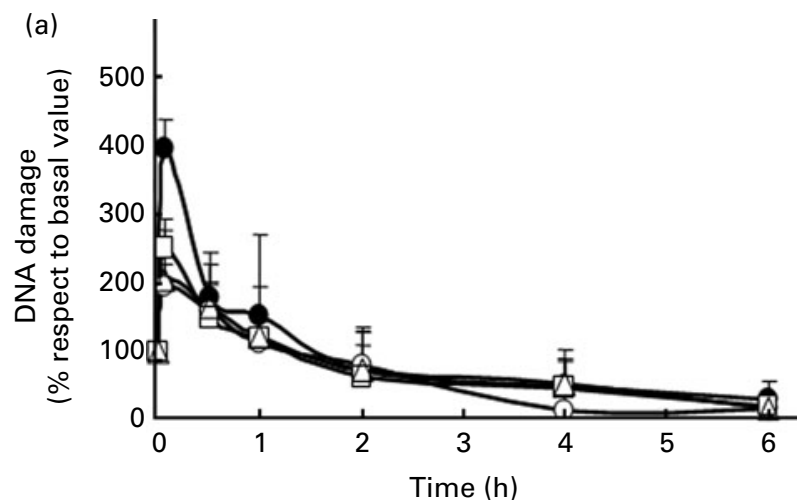

(b)

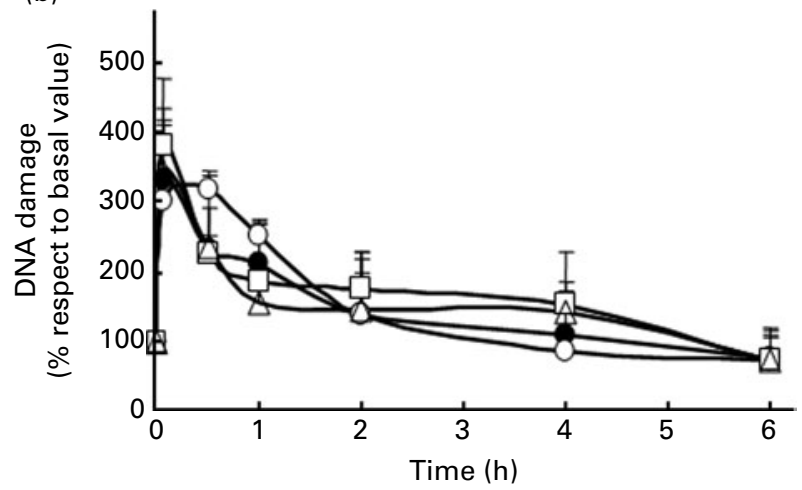

Fig. 2. Kinetics of DNA strand break rejoining in the lymphocytes of dietsupplemented and control subjects undergoing hyperbaric oxygen (HBO) therapy. Lymphocytes isolated from (a) group A ( $n$ 23) and (b) group C ( $n$ 23) subjects at the basal time $(--)$, after the 1 -week diet before $(-\circ)$ and immediately after the $\mathrm{HBO}$ treatment $(-\square-)$ and 1 week after the $\mathrm{HBO}$ treatment $(\triangle-)$ were treated with $\mathrm{H}_{2} \mathrm{O}_{2}$. After oxidation, the cells were suspended in Roswell Park Memorial Institute-1640 medium containing 10\% fetal bovine serum and incubated at $37^{\circ} \mathrm{C}$ for $6 \mathrm{~h}$. At regular interval times $(0,0.5,1,2,4$ and $6 \mathrm{~h})$, aliquots of the samples were collected for DNA strand break analysis. The kinetics of DNA repair were calculated as a percentage of the basal value. Values are means, with standard deviations represented by vertical bars.

\section{Statistical analysis}

Results are expressed as means and standard deviations. Friedman's test for repeated sample was used to evaluate differences among the T1, T2, T3 and T4 time points. Post hoc comparisons were carried out using the Wilcoxon test corrected with the Bonferroni formula to evaluate the differences between baseline (T1) and follow-up (T2, T3 and T4). Differences among the time points of group A $v$. time points of group $\mathrm{C}$ were evaluated by the Kruskal-Wallis test followed by the Mann-Whitney test corrected with the Bonferroni formula. Correlations between AA, plasma HP and TAS were examined by Pearson's correlation analysis. The significance level for all statistical analyses was set at a probability of $<0.05$ (two-tailed test). All data in the present study were analysed by the statistical software Statistical Package for Social Sciences version 15 (SPSS Inc., Chicago, IL, USA).

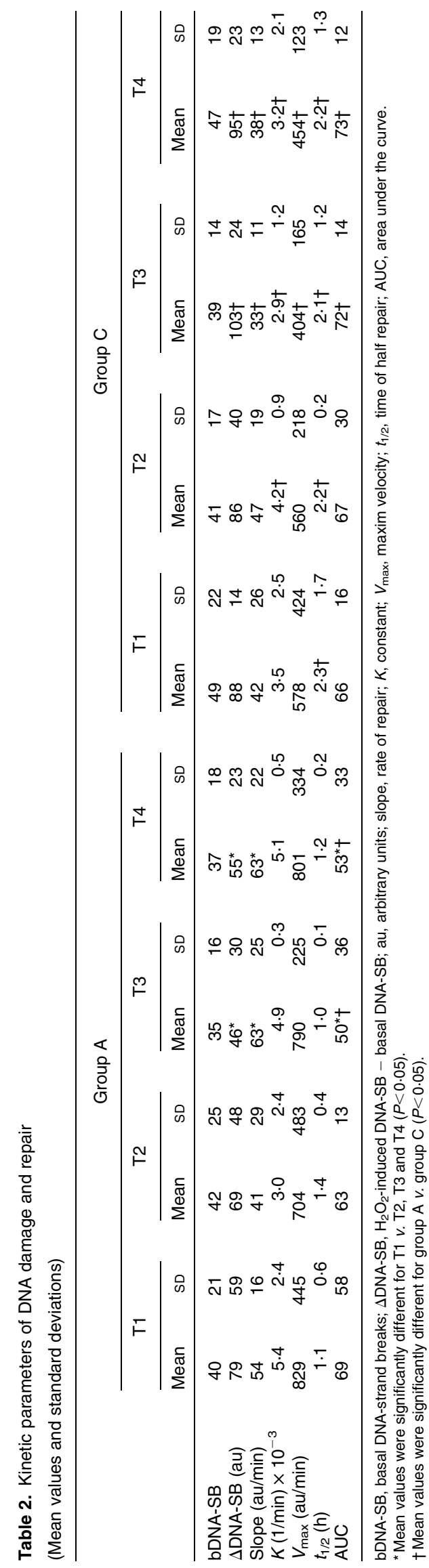




\section{Results}

\section{Effects of ascorbic acid-rich diet intervention on oxidant/ antioxidant status}

The demographic and basic characteristics of the subjects are given in Table 1. There were no differences in anthropometric parameters, antioxidant/oxidant status and body composition between groups $\mathrm{A}$ and $\mathrm{C}$.

A 1-week dietary intake of AA resulted in a significant increment in plasma AA levels by $35 \%(P<0.05)$ in group A (Fig. 1(a)). The HBO treatment did not result in a significant

(a)

T2 T3 T4

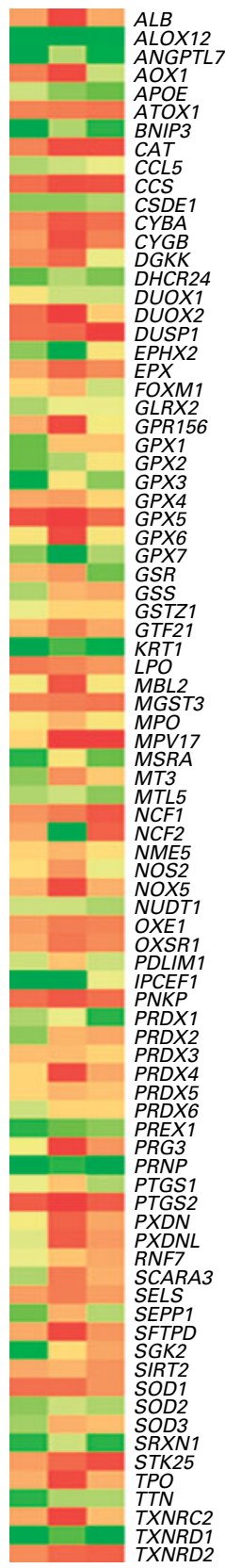

(b)

T2 T3 T4

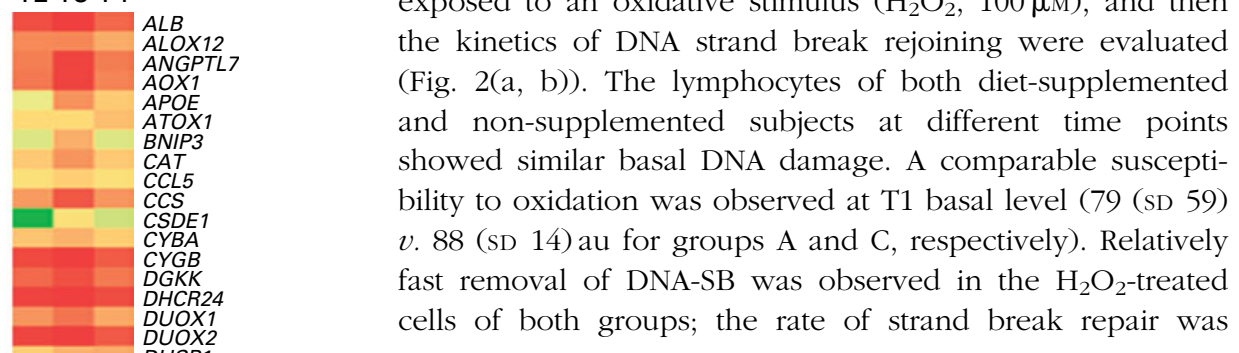
subjects at different time points ( $\mathrm{T} 1, \mathrm{~T} 2, \mathrm{~T} 3$ and $\mathrm{T} 4$ ) were exposed to an oxidative stimulus $\left(\mathrm{H}_{2} \mathrm{O}_{2}, 100 \mu \mathrm{M}\right)$, and then the kinetics of DNA strand break rejoining were evaluated (Fig. 2(a, b)). The lymphocytes of both diet-supplemented and non-supplemented subjects at different time points suscepti- decrease in plasma AA concentration in either group. Conversely, the in vivo oxidative insult induced lipid peroxide formation, which was found in group $\mathrm{C}$ immediately after the HBO treatment (T3), and persisted 1 week after HBO exposure (T4) (Fig. 1(b)). The AA supplementation significantly inhibited HBO-induced HP and enhanced the antioxidant ability of plasma evaluated as TAS. Fig. 1(c) shows a significant increase in TAS in group A compared with group $\mathrm{C}$ at T2, which persisted over time points. A positive correlation was observed between AA and TAS $(R \quad 0 \cdot 46$, $P<0.001)$, and an inverse correlation was found between AA and HP $(R-0.33, P<0.01)$.

To evaluate whether the AA-rich diet might affect DNA damage and its repair, lymphocytes isolated from the enrolled

Fig. 3. Representative gene expression patterns of eighty-eight genes involved in oxidative stress. In five cases for (a) group $C$ and (b) group $A$, the expression of individual genes, collected at the T2, T3 and T4 time points related to samples collected at $\mathrm{T} 1$, was evaluated for the hierarchical clustering analysis. Individual genes are listed vertically. Relative normalised expression for each gene is represented by colour intensity (green, downexpression; yellow, no expression change; red, overexpression; black, gene not detected). T1, blood samples collected at the basal time; T2, blood samples collected after the 1-week diet before the hyperbaric oxygen (HBO) treatment; T3, blood samples collected immediately after the HBO treatment; T4, blood samples collected 1 week after the HBO treatment. $A L B$, albumin; ALOX12, arachidonate 12-1 poxygenase; ANGPTL7, angiopoietin-like 7; $A O X 1$, aldehyde oxidase 1; $A P O E$, apoE; $A T O X 1$, antioxidant protein 1 homologue; BNIP3, BCL2/adenovirus E1B; CAT, catalase; CCL5, chemokine (C-C motif) ligand; CCS, copper chaperone for superoxide dismutase; CSDE1, cold shock domain containing E1; CYBA, cytochrome b-245; CYGB, cytoglobin; DGKK, diacylglycerol kinase, kappa; DHCR24, 24-dehydrocholesterol reductase; DUOX, dual oxidase; DUSP1, dual specificity phosphatase 1; EPHX2, epoxide hydrolase 2; EPX, eosinophil peroxidase; FOXM1, forkhead box M1; GLRX2, glutaredoxin 2; GPR156, G protein-coupled receptor 156; GPX, glutathione peroxidase; GSR, glutathione reductase; GSS, glutathione synthetase; GSTZ1, glutathione transferase zeta 1; GTF2I, general transcription factor II, KRT1, keratin 1; LPO, lactoperoxidase, MBL2, mannose-binding lectin (protein C) 2; MGST3, microsomal glutathione S-transferase; MPO, myeloperoxidase; MPV17, MpV17 mitochondrial inner membrane; MSRA, methionine sulfoxide reductase; MT3, metallothionein 3; MTL5, metallothionein-like 5; NCF, neutrophil cytosolic factor, NME5, non-metastatic cells 5; NOS2, nitric oxide synthase 2; NOX5, NADPH oxidase; NUDT1, nudix (nucleoside diphosphate linked moiety X)-type motif; OXR1, oxidation resistance 1; OXSR1, oxidative stress responsive 1; PDLIMI, PDZ and LIM domain 1; IPCEF1, interaction protein for cytohesin exchange; PNKP, polynucleotide kinase 3'-phosphatase; PRDX, peroxiredoxin; PREX1, phosphatidylinositol-3,4,5-triphosphate-dependent Rac exchange factor 1; PRG3, proteoglycan 3; PRNP, prion protein; PTGS, PG-endoperoxide synthase; PXDN, peroxidasin homolog (drosophila); PXDNL, peroxidasin homolog (drosophila) like RNF7, ring finger protein 7; SCARA3, scavenger receptor class A; SELS, selenoprotein S; SEPP1, selenoprotein P, plasma 1; SFTPD, surfactant protein D; SGK2, serum/glucorticoid regulated kinase 2; SIRT2, sirtuin 2; SOD, superoxide dismutase; SRXN1, sulfiredoxin 1 homolog; STK25, serine/threonine kinase 25; TPO, thyroid peroxidase, TTN, titin; TXNDC2, thioredoxin domain containing; TXNRD, thioredoxin reductase. 


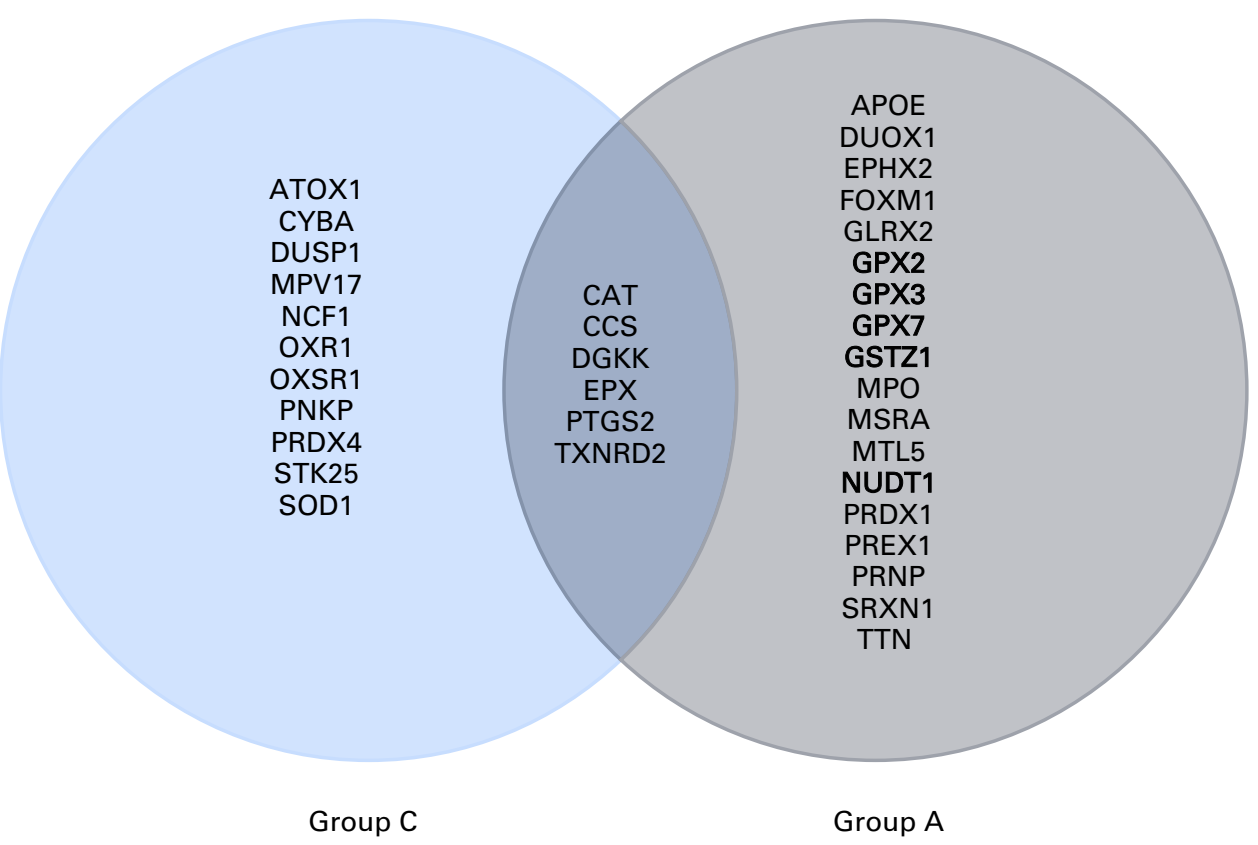

Fig. 4. Venn diagram showing the gene expressed in diet-supplemented and control subjects undergoing hyperbaric oxygen (HBO) therapy. Seventeen genes differing by $>2.0$ - or $<0.5$-fold between samples, collected at the T2, T3 and T4 time points related to samples collected at T1, were overexpressed in group C ( $n 5$, left) after HBO exposure, six of them were also overexpressed in group A ( $n 5$, middle). In addition to these, eighteen genes were exclusively overexpressed in the diet-supplemented group (right). T1, blood samples collected at the basal time; T2, blood samples collected after the 1-week diet before the HBO treatment; T3, blood samples collected immediately after the HBO treatment; T4, blood samples collected 1 week after the HBO treatment. APOE, apoE; ATOX1, antioxidant protein 1 homologue; CAT, catalase; CCS, copper chaperone for superoxide dismutase; CYBA, cytochrome b-245; DGKK, diacylglycerol kinase, kappa; DUOX1, dual oxidase 1; DUSP1, dual specificity phosphatase 1; EPHX2, epoxide hydrolase 2; EPX, eosinophil peroxidase; FOXM1, forkhead box M1; GLRX2, glutaredoxin 2; GPX, glutathione peroxidase; GSTZ1, glutathione transferase zeta 1; MOP, myeloperoxidase; MPV17, MpV17 mitochondrial inner membrane; MSRA, methionine sulfoxide reductase; MTL5, metallothionein-like 5; NCF1, neutrophil cytosolic factor 1; NUDT1, nudix (nucleoside diphosphate linked moiety X)-type motif; OXR1, oxidation resistance 1; OXSR1, oxidative stress responsive 1; PRDX, peroxiredoxin; PNKP, polynucleotide kinase 3'-phosphatase; PREX1, phosphatidylinositol-3,4,5-triphosphate-dependent Rac exchange factor 1; PRNP, prion protein; PTGS, PG-endoperoxide synthase; SOD, superoxide dismutase; SRXN1, sulfiredoxin 1 homolog; STK25, serine/threonine kinase 25; TXNRD, thioredoxin reductase; TTN, titin. The genes significantly overexpressed are shown in bold $(P<0.05)$. (A colour version of this figure can be found online at journals.cambridge.org/bjn).

54 (SD 16) au/min for group A and 42 (SD 26) au/min for group $\mathrm{C}$; the level of DNA-SB formed reached half of the steady-state level within $2 \mathrm{~h}$ after $\mathrm{H}_{2} \mathrm{O}_{2}$ oxidation. Dietary supplementation significantly reduced the levels of $\mathrm{H}_{2} \mathrm{O}_{2}$-induced DNA-SB and accelerated DNA strand break rejoining at T3 and T4 time points. Whereas no changes in DNA repair were found in control subjects. The kinetic parameters of DNA repair are shown in Table 2.

\section{Effects of ascorbic acid-rich diet intervention on gene expression involved in oxidative stress}

To assess whether benefits associated with AA-rich diet consumption might be mediated through changes in the expression of oxidative stress-related genes, a panel of eighty-eight genes involved in ROS detoxification was evaluated in the lymphocytes of five randomly selected dietsupplemented (age 49.8 (SD 8.0); three males, two females) and non-supplemented (age 48.4 (SD 6.0); three males, two females) subjects at different time points. As shown in Fig. 3, oxidative insult induced by the HBO treatment (T3) significantly induces the expression of some oxidative stress-responsive genes such as antioxidant protein 1 homologue (ATOX1), catalase $(C A T)$, cytochrome b-245 (CYBA), copper chaperone for superoxide dismutase (CCS), oxidation resistance 1
(OXR1) and oxidative stress responsive 1 (OXSR1), peroxiredoxin 4 (PRDX4), PG-endoperoxide synthase and cyclo-oxygenase (PTGS2), soluble superoxide dismutase 1 (SOD1) and thioredoxin reductase 2 (TXNRD2) in non-supplemented subjects (group C). Dietary AA intake exclusively induces the expression of important genes for cell detoxification including the GPX family consisting of seven known GPX (1-7). Notably, up-regulation of the glutathione transferase zeta 1 (GSTZ1) gene was found in the AA-rich diet group. This gene is a member of the GST superfamily, which encodes multifunctional enzymes important in the detoxification of electrophilic molecules, including carcinogens, mutagens and several drugs by conjugation with glutathione. In addition, induction of nudix (nucleoside diphosphate linked moiety X)-type motif (NUDT1) gene expression was observed in diet-supplemented subjects. NUDT1 encodes for 7,8-dihydro-8-oxoguanie triphosphatase, which is an enzyme that hydrolyses oxidised purine nucleoside triphosphates, thereby preventing misincorporation. The Venn diagram shows the genes expressed only in groups $\mathrm{A}$ and $\mathrm{C}$ and genes expressed in both groups (Fig. 4).

To evaluate whether the diet-induced gene expression produced an increase in protein expression, GPX and GST protein activities were determined in plasma samples. HBO therapy did not affect GPX activity (T3) in either group, 


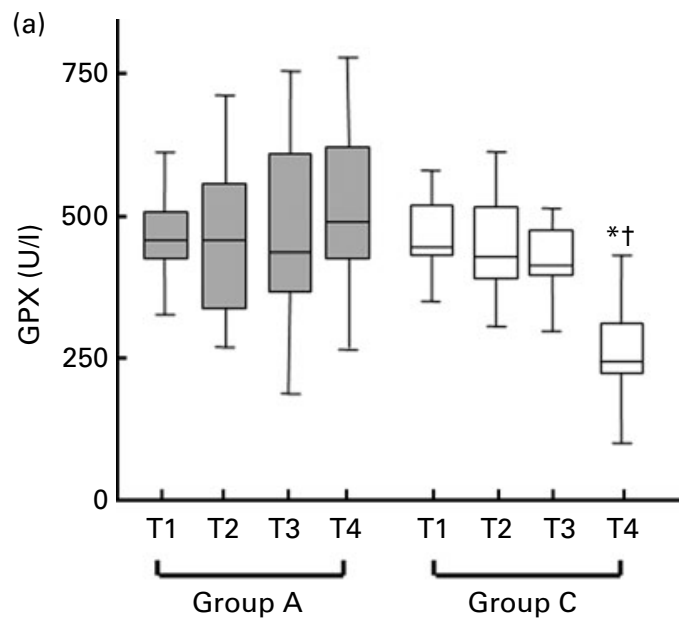

(b)

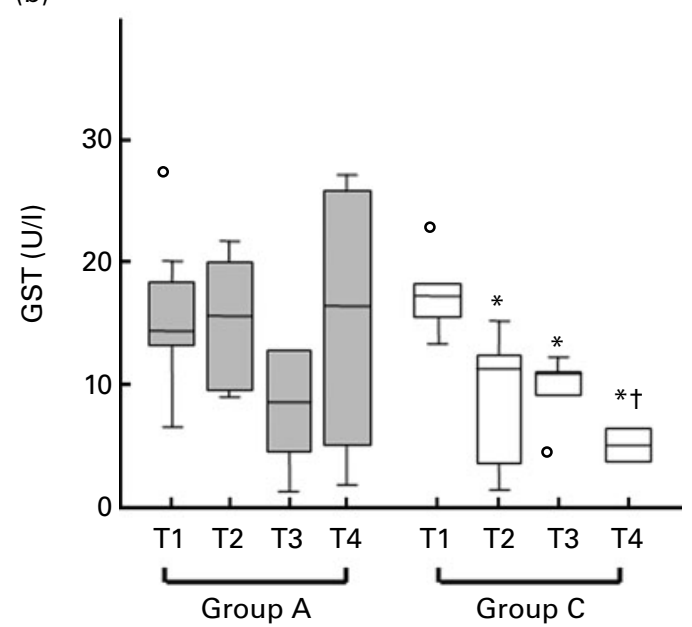

Fig. 5. Box plot showing (a) glutathione peroxidase (GPX) and (b) glutathione S-transferase (GST) activity in the plasma of diet-supplemented and control subjects undergoing hyperbaric oxygen (HBO) therapy. The levels of GPX and GST were assessed in the plasma of diet-supplemented (group A, $n 23$ ) and non-supplemented (group C, $n$ 23) subjects at the basal time (T1), after the 1-week diet before (T2) and immediately after the HBO treatment (T3) and 1 week after the HBO treatment (T4). The lines in the boxes represent the median values. Lines extend to the minimum and maximum values, excluding the outliers $(O)$. * Mean values were significantly different for T1 v. T2, T3 and T4 $(P<0.05)$. † Mean values were significantly different for group A $v$. group $C(P<0.05)$

but a significant decrease in protein activity was found in group C 1 week after the HBO treatment (T4). No decrement of GPX activity was found in the diet-supplemented group (Fig. 5(a)). Similar results were observed for GST plasma activity; a reduced GST activity was found only in the unsupplemented group at the T4 time point (Fig. 5(b)).

\section{Discussion}

The use of large amounts of vegetables and fruits appears to reduce the risk of several important chronic diseases, including CHD and a number of cancers associated with nutritional habits, such as colon, breast and prostate cancer ${ }^{(24-29)}$. Among the nutrients that have 'disease-preventive' properties, vitamins and trace elements have been shown to be the most active components. Several studies and clinic trials, performed to evaluate the effects of vitamin supplementation on oxidative-mediated diseases, have given contradictory results $^{(6-15)}$. In the present study, HBO exposure was used as an in vivo stress model to evaluate the effect of a diet rich in AA (vitamin C) on the increased formation of ROS. $\mathrm{HBO}$ favourably leads to an increase in dissolved $\mathrm{O}_{2}$ in the blood $^{(30)}$. A linear relationship between the formation of $\mathrm{H}_{2} \mathrm{O}_{2}$ and $\mathrm{O}_{2}$ pressure was shown in rats exposed to $\mathrm{HBO}^{(31)}$. Here, volunteers undergoing the HBO treatment were evaluated for oxidative parameters. The intake of AA-rich foods increased antioxidant status and prevented HBO-induced lipid oxidation (Fig. 1).

No DNA-SB formation was observed in the lymphocytes exposed to $\mathrm{HBO}$ either in the control or diet-supplemented group. As reported in our previous study, no DNA-SB formation was found in lymphocytes exposed to $\mathrm{HBO}$, whereas it was found that HBO exposure induced high levels of 8-hydroxy-2'-deoxyguanosine ${ }^{(18)}$. Conversely, Dennog et $a l^{(32)}$ reported the formation of both DNA-SB and 8-hydroxy-2'-deoxyguanosine in the circulating cells of subjects exposed to HBO. The authors have detected DNA damage in the circulating cells directly on the whole blood before the isolation of the lymphocytes. The detection of DNA-SB on isolated lymphocytes used in our studies could explain the discrepancy. DNA-SB are toxic lesions, thus they are quickly repaired. Therefore, DNA-SB induced by HBO exposure could be repaired during the lymphocyte isolation procedure.

To characterise further the antioxidant defence mechanisms, we treated the circulating cells, of diet-supplemented and non-supplemented subjects at different time points, with a ROS production agent $\left(\mathrm{H}_{2} \mathrm{O}_{2}\right)$. With respect to controls, the lymphocytes isolated from diet-supplemented subjects were more resistant to the ex vivo oxidative stimulus and showed an enhanced ability to repair the damaged DNA observed after $\mathrm{HBO}$ exposure, at the $\mathrm{T} 3$ and $\mathrm{T} 4$ time points (Fig. 2; Table 2), suggesting that an adaptive mechanism may be activated. An adaptive protection against the induction of oxidative DNA damage after $\mathrm{HBO}$ has been described previously $^{(18)}$. It has been found that ROS induce various biological processes such as gene expression by stimulating signal transduction components such as $\mathrm{Ca}^{2+}$ signalling and protein phosphorylation ${ }^{(33)}$.

After HBO exposure, circulating cells responded with an increased expression of genes involved in cell detoxification (Fig. 3). Expression of genes encoding for ATOX1, CAT, CYBA, CCS, OXR1 and OXSR1, PRDX4, PTGS2, SOD1 and $T X N R D 2$ increased significantly in response to the insult. Notably, the intake of AA-rich foods induce the expression of other detoxification genes such as the GPX family including GST (Fig. 4). The expression of GPX and GST was enhanced with an increase in the corresponding protein activity found in the AA-rich group 1 week after the HBO treatment (Fig. 5). AA-rich foods may affect the activity of redox-sensitive transcription factors such as Sp1, AP-1 and NF- $\mathrm{BB}$ involved in the expression of distinct detoxification mediators. 
In the light of the recent findings, concepts of antioxidants and redox regulation are being reconsidered, and some new future challenges for redox biology are being outlined ${ }^{(34,35)}$. It has been suggested that antioxidants, thioredoxin-mediated redox regulation and signal transduction mediated by ROS are all part of the same broad mechanism. The integration of different redox inputs, by affecting reversible thiol-disulfide dynamics in a set of target proteins, might result in the regulation of key processes such as proteolysis, gene expression and the functioning of selected metabolic pathways.

Thus, AA-rich foods provide dual protection against oxidative stress; a preventive effect by enhancing plasma antioxidant levels and a detoxification effect by stimulating repair. A preventive effect of antioxidant intake in conditions of oxidative stress is supposed, although a recent study has shown that 4 weeks of supplementation with vitamins $C$ and $\mathrm{E}$ had no preventive effect on $\mathrm{HBO}$-induced oxidative damage. Notably, vitamin supplementation has been found to be associated with a decrement of GSH, suggesting that an imbalance between antioxidants and oxidants contributed to the lack of a preventive effect on oxidative stress ${ }^{(36)}$. It should be pointed out that antioxidant supplementation is not always safe. Although toxicity of consumed antioxidants is very low and only occurs at very high intake levels ${ }^{(37)}$, the degree of benefit or harm of antioxidant supplementation depends also on genetic susceptibilities. Besides that, it is assumed that antioxidant supplements interact with each other and with other dietary constituents affecting the in vivo redox balance. In the present study, the antioxidants were administered by foods such as vegetables. Vegetarian diets are rich in a mixture of antioxidants including phytochemicals. Dietary antioxidants have been thought to be able to modulate cellular responses to various stimuli interacting with ROSmediated intracellular signalling by scavenging ROS or suppressing their generation, either by inhibiting enzymes or by chelating trace elements involved in free radical production, or protecting antioxidant defences or up-regulating intracellular signalling in the antioxidant cellular response ${ }^{(38,39)}$.

In conclusion, by dietary intake, the antioxidants are administrated by a mixture of specific compounds that have been shown to produce an effect or modulate a function in the organism, supporting the use of the nutrigenetic approach to study functional foods.

\section{Acknowledgements}

We thank all the volunteers who participated in the study and the personnel of Hyperbaric Medical Centre of Bologna, Italy. The authors declare that they have no actual or potential competing financial interests, and that there was no funding. R. A. performed the study design, subject enrolment, sample collection and data analysis and manuscript writing. F. D. D. was involved in subject enrolment, HBO therapy treatment and clinical evaluation. E. S., S. S. and L. N. performed the biochemistry and gene expression analyses, data collection and elaboration. B. B. was involved in subject enrolment and clinical evaluation. E. P. performed all statistical analyses. L. S. contributed to the gene expression evaluation and data analysis. M. T. contributed to the biochemistry and gene expression elaboration, data analysis and manuscript writing.

\section{References}

1. Eriksen KT, Sørensen M, Autrup H, et al. (2010) Lifestyle, environmental, and genetic predictors of bulky DNA adducts in a study population nested within a prospective Danish cohort. Toxicol Environ Health 73, 583-595.

2. Kelishadi R, Mirghaffari N, Poursafa P, et al. (2009) Lifestyle and environmental factors associated with inflammation, oxidative stress and insulin resistance in children. Atherosclerosis 203, 311-319.

3. Ignarro LJ, Balestrieri ML \& Napoli C (2007) Nutrition, physical activity, and cardiovascular disease: an update. Cardiovasc Res 73, 326-340.

4. Canoy D, Wareham N, Welch A, et al. (2005) Plasma ascorbic acid concentrations and fat distribution in 19,068 British men and women in the European Prospective Investigation into Cancer and Nutrition Norfolk cohort study. Am J Clin Nutr 82, 1203-1209.

5. Burek CL \& Rose NR (2008) Autoimmune thyroiditis and ROS. Autoimmun Rev 7, 530-537.

6. Bjelakovic G, Nikolova D, Gluud LL, et al. (2007) Antioxidant supplements for primary and secondary prevention: systematic review and meta-analysis. JAMA 297, 842-857.

7. Bjelakovic G, Gluud LL, Nikolova D, et al. (2010) Metaanalysis: antioxidant supplements for liver diseases - the Cochrane Hepato-Biliary Group. Aliment Pharmacol Ther 32, 356-367.

8. Salonen JT, Nyyssönen K, Salonen R, et al. (2000) Antioxidant Supplementation in Atherosclerosis Prevention (ASAP) study: a randomized trial of the effect of vitamins $\mathrm{E}$ and C on 3-year progression of carotid atherosclerosis. J Intern Med 248, 377-386.

9. Kadirvel R, Sundaram K, Mani S, et al. (2007) Supplementation of ascorbic acid and alpha-tocopherol prevents arsenic-induced protein oxidation and DNA damage induced by arsenic in rats. Hum Exp Toxicol 26, 939-946.

10. Wu TC, Huang YC, Hsu SY, et al. (2007) Vitamin E and vitamin C supplementation in patients with chronic obstructive pulmonary disease. Int J Vitam Nutr Res 77, 272-279.

11. Retana-Ugalde R, Casanueva E, Altamirano-Lozano M, et al. (2008) High dosage of ascorbic acid and alpha-tocopherol is not useful for diminishing oxidative stress and DNA damage in healthy elderly adults. Ann Nutr Metab 52, 167-173.

12. Salganik RI (2001) The benefits and hazards of antioxidants: controlling apoptosis and other protective mechanisms in cancer patients and the human population. J Am Coll Nutr 20, 464-472.

13. Block G, Patterson B \& Subar A (1992) Fruit, vegetables and cancer prevention: a review of the epidemiological evidence. Nutr Cancer 18, 1-29.

14. Ames BN, Shigenaga MK \& Hagen TM (1993) Oxidants, antioxidants, and the degenerative diseases of aging. Proc Natl Acad Sci U S A 90, 7915-7922.

15. Jacobson JS, Begg MD, Wang LW, et al. (2000) Effects of a 6 -month vitamin intervention on DNA damage in heavy smokers. Cancer Epidemiol Biomarkers Prev 9, 1303-1311.

16. Kuffler DP (2010) Hyperbaric oxygen therapy: an overview. $J$ Wound Care 19, 77-79.

17. Gröger M, Oter S, Simkova V, et al. (2009) DNA damage after long-term repetitive hyperbaric oxygen exposure. J Appl Physiol 106, 311-315. 
18. Alleva R, Nasole E, Di Donato F, et al. (2005) alpha-Lipoic acid supplementation inhibits oxidative damage, accelerating chronic wound healing in patients undergoing hyperbaric oxygen therapy. Biochem Biophys Res Commun 333, 404-410.

19. Alleva R, Tomasetti M, Sartini D, et al. (2008) alpha-Lipoic acid modulates extracellular matrix and angiogenesis gene expression in non-healing wounds treated with hyperbaric oxygen therapy. Mol Med 14, 175-183.

20. Matsunami T, Sato Y, Sato T, et al. (2009) Oxidative stress and gene expression of antioxidant enzymes in the streptozotocin-induced diabetic rats under hyperbaric oxygen exposure. Int J Clin Exp Pathol 30, 177-188.

21. Tomasetti M, Alleva R, Borghi $\mathrm{B}$, et al. (2001) In vivo supplementation with coenzyme Q10 enhances the recovery of human lymphocytes from oxidative DNA damage. FASEB J 15, 1425-1427.

22. Bessey O, Lowry O \& Brock M (1947) The quantitative determination of ascorbic acid in small amounts of white blood cells and platelets. J Biol Chem 168, 197-205.

23. Vassalle C, Boni C \& Di Cecco P (2006) Automation and validation of a fast method for the assessment of in vivo oxidative stress levels. Clin Chem Lab Med 44, 1372-1375.

24. Paluszczak J, Krajka-Kuźniak V \& Baer-Dubowska W (2010) The effect of dietary polyphenols on the epigenetic regulation of gene expression in MCF7 breast cancer cells. Toxicol Lett 192, 119-125.

25. Miene C, Klenow S, Veeriah S, et al. (2009) Impact of apple polyphenols on GSTT2 gene expression, subsequent protection of DNA and modulation of proliferation using LT97 human colon adenoma cells. Mol Nutr Food Res 53, 1254-1262.

26. Konstantinidou V, Covas MI, Muñoz-Aguayo D, et al. (2010) In vivo nutrigenomic effects of virgin olive oil polyphenols within the frame of the Mediterranean diet: a randomized controlled trial. FASEB J 24, 2546-2557.

27. Jung M, Triebel S, Anke T, et al. (2009) Influence of apple polyphenols on inflammatory gene expression. Mol Nutr Food Res 53, 1263-1280.
28. Weisburger JH (2002) Lycopene and tomato products in health promotion. Exp Biol Med 227, 924-927.

29. Narkowicz CK, Vial JH \& McCartney PW (1993) Hyperbaric oxygen therapy increases free radical levels in the blood of humans. Free Rad Res Commun 19, 71-80.

30. De Martino G, Luchetti M \& De Rosa RC (1996) Toxic effects of oxygen. In Handbook on Hyperbaric Medicine, pp. 59-74 [G Oriani, A Marroni and F Wattel, editors]. New York, NY: Springer-Verlag.

31. Rothfuss A, Dennog C \& Speit G (1998) Adaptive protection against the induction of oxidative DNA damage after hyperbaric oxygen treatment. Carcinogenesis 19, 1913-1917.

32. Dennog C, Hartmann A, Frey G, et al. (1996) Detection of DNA damage after hyperbaric oxygen (HBO) therapy. Mutagenesis 11, 605-609.

33. Suzuki YJ, Forman HJ \& Sevanian A (1997) Oxidants as stimulators of signal transduction. Free Radic Biol Med 22, 269-285.

34. Bourdonnay E, Morzadec C, Fardel O, et al. (2009) Redoxsensitive regulation of gene expression in human primary macrophages exposed to inorganic arsenic. J Cell Biochem 107, 537-547.

35. Calabrese V, Cornelius C, Mancuso C, et al. (2010) Redox homeostasis and cellular stress response in aging and neurodegeneration. Methods Mol Biol 610, 285-308.

36. Beder N, Bosy-Westphal A, Koch A, et al. (2006) Influence of vitamin $\mathrm{C}$ and $\mathrm{E}$ supplementation on oxidative stress induced by hyperbaric oxygen in healthy men. Ann Nutr Metab 50, 173-176.

37. Bendich A \& Machlin LJ (1988) Safety of oral intake of vitamin E. Am J Clin Nutr 48, 612-619.

38. Virgili F \& Marino M (2008) Regulation of cellular signals from nutritional molecules: a specific role for phytochemicals, beyond antioxidant activity. Free Radic Biol Med $\mathbf{4 5}$, 1205-1216.

39. Porrini M (2008) Functional foods: from theory to practice. Int J Vitam Nutr Res 78, 261-268. 